\title{
Phase Behavior of Colloidal Molecular Crystals on Triangular Light Lattices
}

\author{
Matthias Brunner and Clemens Bechinger \\ Physics Department, University of Konstanz, D-78457 Konstanz, Germany
}

(Received 12 February 2002; published 31 May 2002)

\begin{abstract}
We investigate the melting process of a two-dimensional charge-stabilized colloidal system in the presence of a triangular substrate potential. This potential is formed by an optical interference pattern that allows the substrate strength to be varied continuously. By means of an additional scanned optical tweezer the particle density can be adjusted to different numbers $m$ of colloidal particles per substrate minima; here we concentrate on the case of trimers, i.e., $m=3$. Because trimers exhibit additional internal degrees of rotational freedom, the phase behavior of such a system is very different from homogeneous or one-dimensional periodic substrate potentials.
\end{abstract}

DOI: $10.1103 /$ PhysRevLett.88.248302

After several decades of intense scientific work, twodimensional (2D) freezing and melting still remains of high interest to a broad scientific community. This interest is largely motivated by the fundamental predictions within the framework of the Kosterlitz-Thouless-HalperinNelson-Young (KTHNY) theory, i.e., that melting in 2D in contrast to its three-dimensional counterpart - occurs via two sequential defect-driven continuous phase transitions (for a review, see [1]). Given the striking nature of this rather unusual phase transition, it is not surprising that it has triggered many experimental investigations which aimed at testing the KTHNY theory. Among conveniently accessible experimental systems for basic investigations of 2D melting such as liquid crystals [2] and LangmuirBlodgett films [3], colloidal particles deserve particular mention because they allow direct optical observation of topological defects $[4,5]$. While most of the work with 2D colloidal suspensions so far considered only their mutual interactions, real 2D systems do not exist as selfcontained objects but are usually confined to solid (crystalline) or liquid substrates. Accordingly, their phase behavior is closely connected to the properties of the underlying substrate potential and it is the interplay between the particle-particle interactions within the 2D system and the particle-substrate interaction, which leads to very complex phase behavior in such situations [6].

One intriguing example of substrate-induced effects is the observation that silica spheres can crystallize in a square lattice when exposed to a topographically patterned substrate [7]. Alternatively, substrate potentials in colloidal systems can be achieved by means of light forces using interfering laser beams $[8,9]$. Because the geometry of a modulated light field can be easily altered by changing the number and angle of incidence of the laser beams, optical substrate potentials are more convenient than topographical structures for systematic studies of $2 \mathrm{D}$ systems on patterned substrates. Even more important than the high flexibility regarding the geometry of light-induced substrate potentials, however, is the possibility that the substrate strength $V_{0}$ can be varied in situ by changing the laser intensity or the polarization of the laser beams, re-
PACS numbers: 82.70.Dd, 64.70.Dv

spectively $[8,10]$. This is also of high advantage compared to atomic systems where the substrate potential cannot easily be changed.

In this Letter we investigate the case of a colloidal monolayer interacting with a $2 \mathrm{D}$ light potential with triangular symmetry (corresponding to an atomic monolayer on a $\langle 111\rangle$ surface), which was created by interference of three laser beams. Since in our experiments the number density of particles is an integer multiple $m=3$ of the number density of potential substrate minima, trimers are localized as loosely bound aggregates (colloidal molecules) in each potential well. Upon increasing the substrate strength $V_{0}$ a colloidal molecular crystal with high positional and orientational order of the trimers is observed. Further enhancement of $V_{0}$ leads to a loss of orientational order. Our results are in qualitative agreement with recent results of Langevin simulations, where the melting behavior for dimers and trimers was investigated [11]. Because an $m$-mer can be considered as a single particle with internal degrees of rotational freedom, the melting process of this novel colloidal crystalline state is very different compared to that of homogeneous substrates and may lead to a better understanding of the phase behavior of molecules on crystalline surfaces.

For the colloidal system we used aqueous suspensions of highly charged sulphate-terminated polystyrene particles of $3 \mu \mathrm{m}$ diameter from Interfacial Dynamics Corporation with an average polydispersity below $4 \%$. The pair interaction of particles can be described by a screened Coulomb potential $[12,13]$. As the sample cell we used a silica glass cuvette with $1 \mathrm{~mm}$ spacing, which was connected to a deionization circuit to control the salt concentration of the suspension. This circuit consisted of the sample cell, an electrical conductivity meter, a vessel of ion exchange resin, a reservoir basin, and a peristaltic pump [14]. Before each measurement, the water was pumped through the ion exchanger, and typical ionic conductivities below $0.07 \mu \mathrm{S} / \mathrm{cm}$ were obtained. Afterwards the colloidal suspension was injected into the cell, which was then disconnected from the circuit during the measurements. This procedure yielded stable and reproducible ionic conditions over many hours. To characterize the particle interaction 
strength, we determined the Debye screening length $\kappa^{-1}$ by analyzing the pair correlation function $g(r)$ of the homogeneous 2D liquid phase via a deconvolution of the Ornstein-Zernike equation [15]. For all measurements presented here, $\kappa^{-1}$ was about $570 \mathrm{~nm}$.

The light potential was formed by three beams of a linearly polarized $\mathrm{Nd}: \mathrm{YVO}_{4}$ laser $\left(\lambda=532 \mathrm{~nm}, P_{\max }=\right.$ $5 \mathrm{~W}$ ) which intersected at an angle of $60^{\circ}$ in the sample plane, where they formed a triangular interference pattern, as shown in Fig. 1a. Because of light forces, the particles experience a substrate potential whose symmetry corresponds to that of the underlying light intensity distribution. The strength of the potential was determined using the particle density distribution of a highly diluted colloidal system. The vertical light pressure of the three beams forces the negatively charged particles to the equally charged glass surface of our sample cell and reduces vertical particle fluctuations to less than $100 \mathrm{~nm}$. Accordingly, the system can be considered as 2D to good approximation.

The particle density was adjusted by a scanned optical tweezer which acted as a boundary box for the investigated 2D colloidal suspension. The beam of an argon ion laser $(\lambda=488 \mathrm{~nm}, P=0.4 \mathrm{~W})$ was reflected from a $2 \mathrm{D}$ galvanostatically driven mirror and focused with a spot diameter of about $2 \mu \mathrm{m}$ onto the sample plane. The mirror was controlled by a computer in order to scan a quadratic contour with $200 \mu \mathrm{m}$ side length in the central region of the interference pattern. The repetition rate of the pattern was on the order of $50 \mathrm{~Hz}$, therefore we obtained a quasistatic optical trap with a depth of about $30 k_{B} T$ along the contour of the box. Accordingly, the particles ordered in a 1D dense packing along the contour of the laser trap and thus served - due to their electrostatic interaction - as a corral for the particles inside the boundary box. Slow variation of the box size thus yields a convenient way to precisely adjust the particle density inside the box as this is essential to our experiments [15]. We adjusted the number density of colloids to be commensurate with the underlying light lattice, i.e., the number of particles to be an integer multiple $m$ of the substrate lattice. Here we will concentrate on the

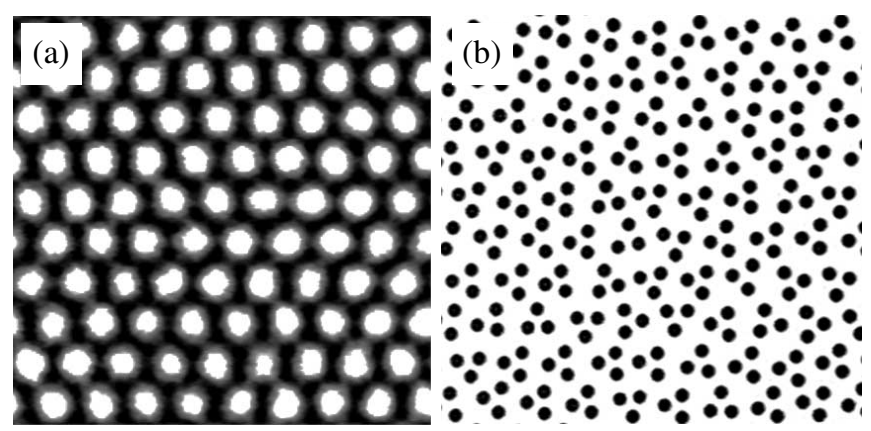

FIG. 1. Optical images of (a) the triangular optical interference pattern which serves as the substrate potential and (b) colloidal particles which form trimers at sufficiently high substrate strengths. The region corresponds to about $100 \times 100 \mu \mathrm{m}^{2}$. case $m=3$ corresponding to three particles per potential minimum. As is seen in Fig. 1b, this leads to the localization of trimers on each laser lattice site for sufficiently high laser fields and demonstrates that the laser field acts as a surface potential for the particles.

In order to investigate the effect of the substrate potential depth on the structure of the colloidal system, we varied the laser intensity and determined the spatially resolved average particle density $\rho(x, y)$ in a central region of the interference pattern. In addition, we also calculated the averaged local particle density in a substrate potential well $\rho_{\text {loc }}(x, y)$. Both quantities are plotted as the left and right columns of Fig. 2. During the experiments the particle density was adjusted to about $5 \%$ below the critical density required for spontaneous crystallization. Accordingly, the system is in a liquid state when no 2D light lattice is applied, as shown in Figs. 2a and 2b. When the laser field is turned on, however, considerable changes in the structure of the 2D colloidal system are observed, as will be discussed below. Figures $2 \mathrm{c}-2 \mathrm{~h}$ show contour plots of $\rho(x, y)$ and $\rho_{\text {loc }}$ for three different laser intensities, corresponding to substrate potentials $V_{0}$ of $40 k_{B} T$, $60 k_{B} T$, and $110 k_{B} T$, respectively, where $k_{B} T$ is the thermal energy at room temperature. Figure 2 was obtained by analyzing more than 1000 pictures with $1 \mathrm{~s}$ time interval each. At $V_{0}=40 k_{B} T$ (Figs. 2c and 2d) the density distribution is no longer homogeneous because the particles start to interact with the substrate potential. At these moderate substrate strengths, however, most of the particles are able to surmount the barriers between the substrate potential wells and only a few particles become trapped in confined regions. Accordingly, the corresponding plot of $\rho_{\text {loc }}$ shows a relatively broad particle distribution. Upon increasing the substrate strength the localization of particles increases and at $V_{0}=60 k_{B} T$ essentially all of the potential wells are occupied with trimers (Fig. 2e). As seen in Fig. 2f, these trimers also possess a very high degree of orientational order. The occurrence of such a crystalline phase with both positional and orientational order is also confirmed by numerical simulations [11]. In addition to the three most pronounced spots which indicate the main orientation of the trimers, Fig. $2 \mathrm{f}$ shows another three weak spots which correspond to defects where the orientation of trimers is rotated by $60^{\circ}$. These two orientations correspond to the twofold degenerate ground state of the system [11]. When crystallization started, we initially observed regions with both trimer orientations until finally one orientation grew at the expense of the other. In some experiments we also found stable grain boundaries between crystallites having different trimer orientation which suggests that orientational freezing is of first order.

When the substrate strength is further increased to $110 k_{B} T$, the centers of the trimers become more localized at the lattice sites of the interference pattern. In contrast to the enhanced positional order, however, the orientational 

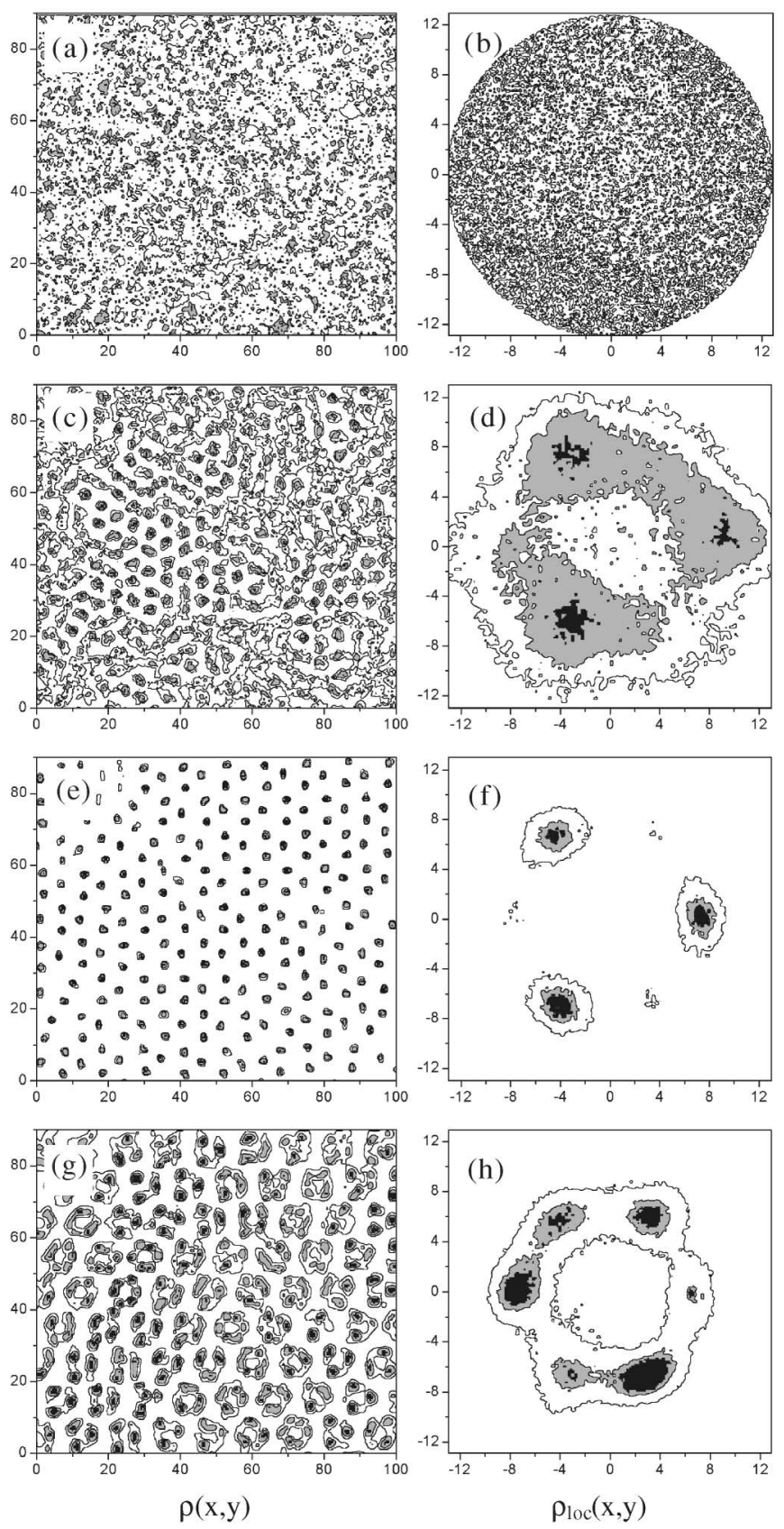

FIG. 2. Contour plots of the lateral density distribution $\rho$ and the averaged local particle density $\rho_{\text {loc }}$ for different light potentials $V_{0}$ : (a),(b) $0 k_{B} T$, (c),(d) $40 k_{B} T$, (e),(f) $60 k_{B} T$, and (g),(h) $110 k_{B} T$. The horizontal axes are $x$ and $y$, respectively. All units are in $\mu \mathrm{m}$.

order of the trimers becomes much weaker as seen from the ring-shaped density distribution in Fig. $2 \mathrm{~g}$. This is supported by Fig. $2 \mathrm{~h}$ which shows that the orientation of the trimers is more diffuse in angular direction. These results clearly demonstrate that increasing the depth of the potential wells leads to a loss of orientational order between the trimers.

The orientational melting can be analyzed in a quantitative way by calculating the order parameter for the orien- tational order of the trimers,

$$
\Psi_{k}=\exp \left[3 i \phi_{k}\right]
$$

where $\phi_{k}$ is the orientation of the vector between a particle and the center of the corresponding substrate potential well with respect to some fixed reference axis. Similar to the bond orientational correlation function in isotropic 2D systems [1], here we define the orientational correlation function of the trimers,

$$
g_{3}\left(r_{k l}\right)=\operatorname{Re}\left\{\left\langle\Psi_{k}^{*} \Psi_{l}\right\rangle\right\},
$$

with the brackets corresponding to summation over all particles forming the trimers. With this definition, perfect orientational order of the trimers corresponds to $g_{3}(r)=1$ while perfect anticorrelation yields $g_{3}(r)=-1$. For $V_{0}=$ $40 k_{B} T$ the orientational correlation between the trimers is short ranged and decays almost to zero after $25 \mu \mathrm{m}$ (corresponding to about two lattice periods) reflecting total loss of orientational order. At $V_{0}=60 k_{B} T$ the decay of $g_{3}(r)$ occurs on much longer length scales and even after ten lattice periods $g_{3}(r)$ corresponds to about $25 \%$ of its initial value (Fig. 3b). At the highest substrate strength $\left(V_{0}=110 k_{B} T\right)$ the decay is much faster again (Fig. 3c). This is in agreement with the orientational remelting of the trimers as observed in Figs. $2 \mathrm{~g}$ and $2 \mathrm{~h}$.

To understand why the coupling between the trimers is lost at high potential strengths, one has to consider two mechanisms of similar importance: On the one hand, a stronger substrate potential increases the radial pressure on the trimers and therefore reduces their mean extent. This results in larger distances between particles of different trimers. Because of the exponential distance dependence of the screened Coulomb potential, this leads to a reduction of the trimer interaction when $V_{0}$ is increased. On the other hand, the colloidal particles are subjected to thermal fluctuations which amount in the crystalline phase to about 50\% of the interparticle distance. The radial fluctuations of the particles inside the trimers contribute to their interaction strength. This becomes most important in the crystalline phase where those fluctuations lead to an angular registration between trimers, i.e., to long-range orientational order. When $V_{0}$ is increased, both the lateral fluctuations and the mean lateral extent of the trimers decrease which reduces the coupling between adjacent trimers and thus leads to orientational melting.

The relationship between radial and angular fluctuations can be estimated from the orientational correlation function at distance zero, i.e., $g_{3}(0)$, which corresponds to the selfcorrelation of the trimers. The deviation of $g_{3}(0)$ from 1 characterizes to what extent thermal fluctuations cause an angular deviation of the trimers from an ideal equilateral triangle. As is seen in Figs. $3 b$ and $3 c, g_{3}(0)$ at $60 k_{B} T$ exceeds the corresponding value at $110 k_{B} T$ by about $20 \%$. This means that, although the localization of trimers inside 


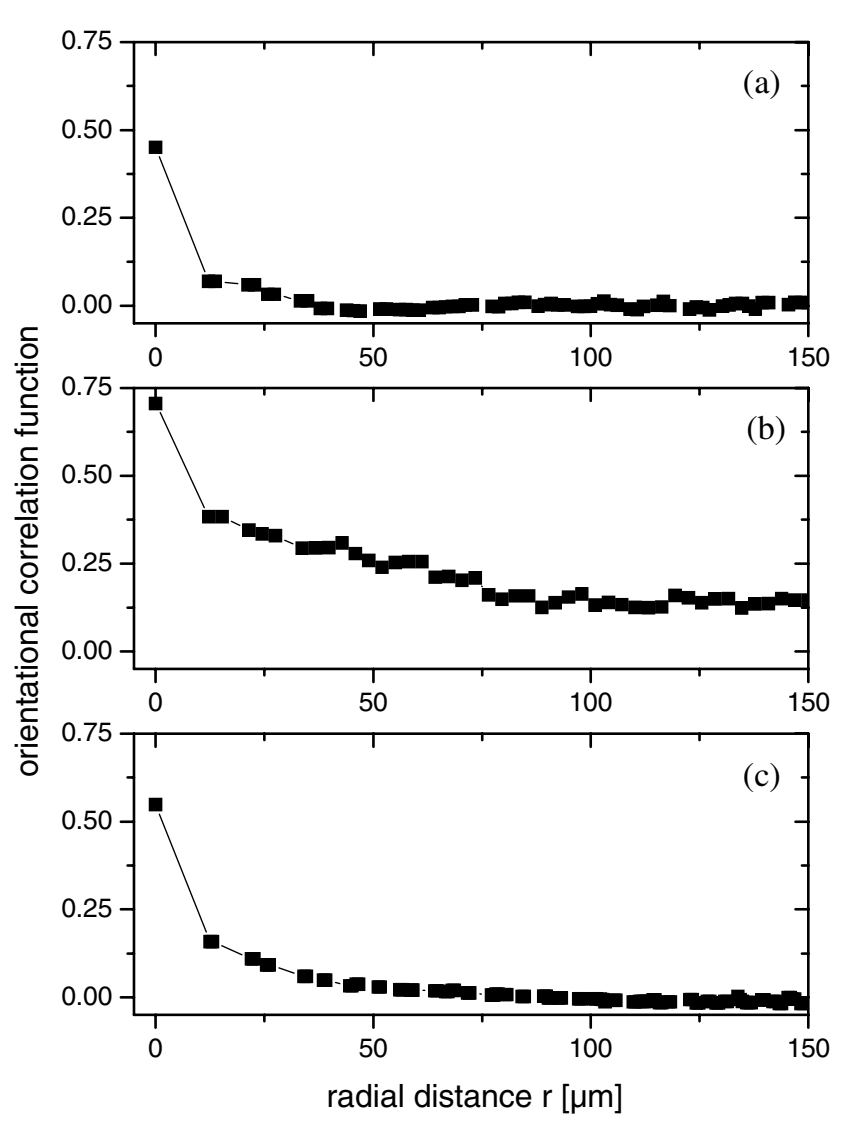

FIG. 3. Averaged orientational correlation function $g_{3}(r)$ for (a) $V_{0}=40 k_{B} T$, (b) $60 k_{B} T$, and (c) $110 k_{B} T$.

the potential wells increases at higher substrate strength, a stronger deviation in the angular shape of the trimers occurs. This strongly supports the hypothesis mentioned above that the reduction of the radial fluctuation leads to an increase in the angular fluctuation in trimers.

We hope that this work will stimulate further studies on the melting of colloidal particles in periodic 2D substrate potentials. In particular, it would be interesting to compare the decay of the orientational correlation function with theoretical predictions.

In summary, we have studied the phase behavior of a charge-stabilized 2D colloidal suspension subjected to a periodic, 2D optical substrate potential. The number of colloidal particles was adjusted by a computer controlled scanned optical tweezer to be 3 times as high as the num- ber of substrate potential wells. Upon gradually increasing the substrate potential the colloidal particles first start to become localized as trimers in the potential wells. At intermediate potential values the system responds with long-range orientational and positional order, and at higher potential strengths the orientational order is lost, leaving the trimers to rotate in the potential wells. This phase behavior is qualitatively explained in terms of particle fluctuations.

We thank C. Reichhardt for helpful discussions. The work was supported by the Deutsche Forschungsgemeinschaft under Grant No. Be1788/3.

[1] D. R. Nelson, Phase Transitions and Critical Phenomena (Academic, London, 1983).

[2] C. C. Huang, in Bond-Orientational Order in Condensed Matter Systems, edited by K. J. Strandburg (Springer, New York, 1992).

[3] C. Knobler, Annu. Rev. Phys. Chem. 43, 207 (1992).

[4] C. A. Murray, W. O. Springer, and R. A. Wenk, Phys. Rev. B 42, 688 (1990).

[5] A. Pertsinidis and X. S. Ling, Phys. Rev. Lett. 87, 098303 (2001); A. Pertsinidis and X. S. Ling, Nature (London) 413, 147 (2001).

[6] S. N. Coppersmith, D. S. Fisher, B. I. Halperin, P. A. Lee, and W. F. Brinkmann, Phys. Rev. B 25, 349 (1982).

[7] A. v. Blaaderen, R. Ruel, and P. Wiltzius, Nature (London) 385, 321 (1997).

[8] A. Chowdhury, B. J. Ackerson, and N. A. Clark, Phys. Rev. Lett. 55, 833 (1985).

[9] K. Loudiyi and B. J. Ackerson, Physica (Amsterdam) 184A, 1 (1992).

[10] C. Bechinger, M. Brunner, and P. Leiderer, Phys. Rev. Lett. 86, 930 (2001).

[11] C. Reichhardt and C. J. Olson, preceding Letter, Phys. Rev. Lett. 88, 248301 (2002).

[12] B. V. Derjaguin and L. Landau, Acta Physicochim. URSS 14, 633 (1941).

[13] E. J. W. Vervey and J.T.G. Overbeek, Theory of the Stability of Lyophobic Colloids (Elsevier, Amsterdam, 1948).

[14] T. Palberg, W. Härtl, U. Wittig, H. Versmold, M. Würth, and E. Simnacher, J. Phys. Chem. 96, 8180 (1992).

[15] M. Brunner, C. Bechinger, W. Strepp, V. Lobaskin, and H. H. v. Grünberg, Europhys. Lett. (to be published). 